

\section{Sumário}

O USO DE BOTS SOCIAIS COMO AMEAÇA À DEMOCRACIA .............................................................13

Mateus de Oliveira Fornasier

In Memoriam: The Republican Form and the Separation-of-Powers Among the Four

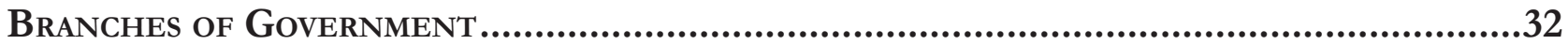

Farris Lee Francis

TODOS E CADA UM DE NÓs: O INTERESSE PÚBLICO COMO CRITÉRIO DE DESENVOLVIMENTO HUMANO

Mártin Haeberlin e Flávio Comim

FACTORES DERIVAdos DE LA POBREZA MULTIDIMENSIONAL QUE AFECTAN LA USABILIDAD DEL e-gobierno en México

Oscar Yahevh Carrera Mora, Luis Fernando Villafuerte e Saulo Sinforoso Martínez

¿Qué ha PASAdo CON Los PRINCIPIOS DE Universalidad, Solidaridad y EFICIENCIA DEL Sistema General de Seguridad Social en Salud de Colombia?

David Mendieta e Carmen Elena

CHANGING THE BENCH FOR A HANDSHAKE: LITIGATION, ADMINISTRATIVE RESOLUTION AND MEDIATION IN FREEDOM OF INFORMATION COMPLAINTS IN CHILE

Pablo Contreras

A evolução identitária da Controladoria-Geral da União: polifonia e decisões em POLÍTICAS DE TRANSPARÊNCIA E ACESSO À INFORMAÇÃO

Érica Bezerra Queiroz Ribeiro e Bruno Amaral Machado

As RAÍZES CRISTÃS DO PRINCÍPIO JURÍDICO DA FRATERNIDADE E AS CRISES MIGRATÓRIAS DO TERCEIRO MILÊNIO.

Maria Celeste Cordeiro Leite dos Santos e Marilene Araujo

JUDICIALIZAÇÃo DESCENTRALIZADA E INDIVIDUALIZADA DA POLÍTICA: MUDANÇAS NAS REGRAS DE tramitação de Medidas Provisórias a partir da Emenda Constitucional 32 155 Leandro Molhano Ribeiro e Mariana Novotny Muniz

O PAPEL DO CNJ DIANTE DO RECONHECIMENTO DO ESTADO DE COISAS INCONSTITUCIONAL DO SISTEMA CARCERÁRIO BRASILEIRO NA PERSPECTIVA DO ATIVISMO DIALÓGICO. 176 Ana Paula Kosak e Estefânia Maria de Queiroz Barboza 
Efeitos do Programa Mais Médicos (PMM) nos resultados da Atenção Básica À SAÚDE.

Alex dos Santos Macedo e Marco Aurélio Marques Ferreira

OrÇamento para os direitos das CRianÇas E adolescentes EM CuRitiba: Plano E EXECUÇÃO

Karoline Strapasson Jambersi e Antonio Gonçalves de Oliveira

A Capacidade do Estado frente a gestão de riscos e desastres após a Política Nacional de Proteção e Defesa Civil (Lei 12.608/2012) .............................................245

Larissa Maria da Silva Ferentz e Carlos Mello Garcias

REFunCIONALIZAÇÃo da PENA DE PRISÃo: ABORDAGEM ACERCA DA ALIENAÇÃo DO TRABALHO

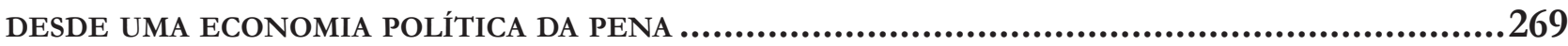
Jackson da Silva Leal

LA CONSTITUCIONALIZACIÓN DEL DERECHO A DEFENSA JURIDICA DE LAS VICTIMAS EN CHILE 286 Marcela Peredo Rojas

COLABORAÇÃo PREMIADA E SELETIVIDADE DO SISTEMA PENAL: PROBLEMATIZAÇõES ACERCA DA UTILIZAÇÃo de aCordos Na Operação LaVa Jato

Maiquel Ângelo Dezordi Wermuth e Maurício Habckost Dalla Zen

Poder, MASCULINIDAde E PARTICIPAÇão EM FaCções CRIMINOSAS A PARTIR DE RELATOS ADOLESCENTES PRIVADOS DE LIBERDADE PELA PRÁTICA DE ATOS INFRACIONAIS

Jailson Alves Nogueira, Ramon Rebouças Nolasco de Oliveira, Lauro Gurgel de Brito e Veruska Sayonara de Góis

MobILIZAÇÃo JURÍDICA E O DIREITO AO ABORTO NO BRASIL: A EVOLUÇão ARGUMENTATIVA NAS RESPECTIVAS AÇÕES DE CONTROLE CONCENTRADO DE CONSTITUCIONALIDADE .355

Fabiano Hartmann Peixoto e Thales Alessandro Dias Pereira 


\title{
Factores derivados de la pobreza multidimensional que afectan la usabilidad del e-gobierno en México*
}

\author{
Factors derived from multidimensional \\ poverty that affect the usability of \\ e-government in Mexico
}

\author{
Oscar Yahevh Carrera Mora ** \\ Luis Fernando Villafuerte ${ }^{* * *}$ \\ Saulo Sinforoso Martínez ${ }^{* * * *}$
}

* Recebido em 03/04/2020

Aprovado em 20/04/2020

** Licenciado en Administración, Licenciado en Sistemas Computacionales Administrativos, Maestro en Alta Dirección y Doctor en Ciencias Administrativas y Gestión para el Desarrollo. Miembro del Sistema Nacional de Investigadores. Profesor de la Facultad de Negocios y Tecnologías sede Ixtaczoquitlán de la Universidad Veracruzana. E-mail: ocarrera@uv.mx

*** Doctor en Ciencias Politicas por la Universidad Autonoma de Madrid, Espana. Docente de la Universidad Veracruzana Facultad de Ciencias Administrativas y Sociales, sede Xalapa, Veracruz, México. Miembro del Sistema Nacional de Investigadores.

E-mail: lvillafuerte@uv.mx

**** Doctor en Ciencias Administrativas y Gestión para el Desarrollo. Miembro del Sistema Nacional de Investigadores. Docente de la Facultad de Contaduría sede Tuxpan de la Universidad Veracruzana.

E-mail: ssinforoso@uv.mx

\section{Resumen}

Este análisis tiene como objetivo comprender que factores emanados de la pobreza multidimensional tienen un efecto en la usabilidad de los servicios de e-gobierno. Por lo tanto, se clasificaron los indicadores de la pobreza multidimensional a través de las dimensiones de éxito del e-gobierno (infraestructura e infoestructura), construyéndose un diagrama causal con los indicadores identificados en esta revisión como causales del incremento o decremento en el uso del e-gobierno convirtiéndolos en variables explicativas a través de la metodología de dinámica de sistemas, lo cual pueda facilitar el análisis de escenarios previos al diseño y/o ejecución de una política pública para favorecer la implementación del e-gobierno. La originalidad del estudio radica en el análisis de la pobreza que es un elemento causal para el surgimiento de factores que inhabilitan a la ciudadanía a hacer uso de los servicios públicos electrónicos, la pobreza regularmente es abordada en materia de TIC como un elemento que de facto genera exclusión, pero no solo se trata de señalar a la pobreza como un fenómeno de exclusión y decidirse por visualizar otros escenarios, debido a que la definición de factores que emanan de esta situación podría permitir el diseño e implementación de políticas y estrategias que habiliten a la ciudadanía a utilizar los servicios de e-gobierno. Por lo tanto, se concluye en el desarrollo de un diagrama causal que muestra diferentes factores de la infraestructura e infoestructura que van limitando la usabilidad de los servicios públicos brindados a través de plataformas web (e-gobierno).

Palabras clave: Ciudadanía. e-gobierno. Pobreza. Usabilidad.

\section{Abstract}

This analysis aims to understand which factors arising from multidimensional poverty have an effect on the usability of e-government services. Therefore, the indicators of multidimensional poverty were classified through the dimensions of success of e-government (infrastructure and infostructure), 
constructing a causal diagram with the indicators identified in this review as the causes of the increase or decrease in the use of e-government, converting them into explanatory variables through the system dynamics methodology, which can facilitate the analysis of scenarios prior to the design and/or execution of a public policy to favour the implementation of e-government. The originality of the study lies in the analysis of poverty, which is a causal element for the emergence of factors that disqualify citizens from making use of electronic public services. Poverty is regularly addressed in the area of ICTs as an element that de facto generates exclusion, but it is not only a matter of pointing to poverty as a phenomenon of exclusion and deciding to visualize other scenarios, since the definition of factors that emanate from this situation could enable the design and implementation of policies and strategies that enable citizens to use e-government services. Therefore, we conclude by developing a causal diagram that shows different infrastructure and infostructure factors that limit the usability of public services provided through web platforms (e-government).

Keywords: Citizenship. e-government. Poverty. Usability.

\section{Introducción}

Las reformas a la administración pública que se iniciaron a finales del siglo XX con el proceso de transformación a la Nueva Gestión Pública (NGP), tuvieron como objetivo mejorar el proceso de gestión pública, de donde se pusieron en boga herramientas de gestión tales como Presupuestos Basados en Resultados (PBR), Programas Operativos Anuales (POA), tableros de mando, por solo citar algunos ejemplos, y dentro de estas estrategias se empezó a implementar al mismo tiempo, la aplicación de las herramientas tecnológicas a los procesos administrativos, en donde se utilizaron para diversas áreas de la administración pública, pero básicamente se orientaron en esta primer momento en aspectos financieros de la Administración.

Sin embargo, si es necesario decir, que de estas herramientas de la NGP muy pocas sobrevivieron, las causas de su erosionamiento son varias, pero básicamente, su virtud, que era el establecer un gran número de indicadores, se convirtió en su debilidad, la numeralia terminó volviendo muy complicada a la gestión, los resultados a veces dejaban de ser importantes y se concentraban en cumplir los criterios de planeación, por lo que estas metodologías poco a poco se fueron abandonando, por ejemplo los POA, los tableros de mando, etcétera, sin embargo, el componente que si tuvo una muy buena receptividad tanto institucional como social, fue el uso de las tecnologías de información, el cual permitió implementar el e- gobierno. Su uso se empezó a extender de las áreas financieras como ya se dijo, a las de implementarlas en el ciclo de las políticas públicas, espacio donde tiene una presencia muy fuerte y constante el uso de esta herramienta de la gestión pública.

Así, el e-gobierno se ha convertido en los últimos años en una herramienta muy importante para brindar servicios públicos eficientes para la ciudadanía, por lo cual los gobiernos tanto a nivel nacional como estatal y municipal han venido buscando alternativas para digitalizar los servicios públicos y ofrecerlos a través de plataformas web con tres objetivos en particular, primero, mejorar la comunicación entre ciudadano-gobierno (inclusión), segundo, agilizar los trámites administrativos (eficiencia) y c) reducir la corrupción (transparencia).

Por lo cual, se han impulsado diversas estrategias de construcción e implementación de plataformas web de e-gobierno en los diferentes niveles de gobierno, pero la realidad es que los servicios de e-gobierno ofrecidos aún son pocos, así mismo, la usabilidad de los existentes es limitada, en México solo el 31\% de los usuarios utilizan el internet para acceder a servicios gubernamentales ${ }^{1}$, a su vez, se tiene de manifiesto que existe una baja usabilidad de las plataformas existentes de e-gobierno en $\mathrm{México}^{2}$, principalmente las desarrolladas a nivel local.

MÉXICO. Instituto Nacional de Estadística y Geografía (INEGI). 2018.

MÉXICO. Instituto Méxicano para la Competitividad A.C. (IMCO). 2019. 
Existen diferentes respuestas a esa problemática, una de ellas es que una parte de la población son menores de 18 años y no requieren hacer uso de los servicios y otra parte de la población son personas de la tercera edad que no se han podido adaptar a esos nuevos mecanismos, pero también existen otras respuestas que se han definido como factores que afectan la usabilidad del e-gobierno como lo son: la falta de conocimientos que tienen los ciudadanos para el uso de las computadoras personales, el analfabetismo, el analfabetismo digital, la desconfianza de la ciudadanía de los servicios públicos ofrecidos a través de internet derivado de una percepción de eficiencia negativa ${ }^{3}$ y la brecha digital ${ }^{4}$.

Sin embargo, estos factores mencionados y otros más, tienen un origen, por lo cual el planteamiento central de este estudio considera que la pobreza es un elemento causal para el surgimiento de esos factores que inhabilitan a la ciudadanía a hacer uso de los servicios públicos electrónicos, y es que la pobreza regularmente es abordada en materia de TIC (Tecnologías de Información y Comunicación) como un elemento que de facto genera exclusión, barreras digitales, entre otros elementos implícitos, pero en este estudio no solo se trata de señalar a la pobreza como un fenómeno de exclusión que es restrictivo del uso de las TIC y decidirse por visualizar otros escenarios, de hecho la relación entre la pobreza y la usabilidad del e-gobierno ha sido abordada por diferentes estudios ${ }^{5}$, pero como un elemento per se o incluso se señala al e-gobierno como un mecanismo que pudiese reducir la pobreza ${ }^{7}$, por lo tanto, los estudios se limitan a definir a la pobreza como un factor total de exclusión de la ciudadanía sobre las TIC que esta implícito sin entrar a detalle al conocimiento de cada unas de las variables que se manifiestan.

La propuesta de este estudio radica en abordar como estas condicionantes definidas como variables o factores que surgen de una condición de pobreza tienen una causalidad específica sobre la usabilidad del e-gobierno que necesita hacerse explicita, acción que pudiera permitir a los hacedores de políticas públicas tener una noción más clara de todos los factores y variables que pueden surgir como una limitante en la usabilidad de las TIC, posterior a la implementación de estos mecanismos, por lo que, la definición de factores que emanan de una situación de pobreza podría permitir el diseño e implementación de políticas y estrategias que habiliten a la ciudadanía en esa condición a utilizar los servicios de e-gobierno, pudiendo con ello contar con elementos tangibles para aumentar la usabilidad, haciendo exitoso al e-gobierno en materia de inclusión de la ciudadanía, involucrando a esos sectores sociales vulnerables.

En consecuencia, el objetivo de esta revisión es establecer que factores emanados de la pobreza tienen un efecto en la usabilidad de los servicios públicos brindados a través de plataformas web, lo cual permita a partir de una visión metodológica basada en el enfoque sistémico focalizar de manera cualitativa una correlación que parece simple entre variables que apunten hacia una usabilidad social y no instrumental de la tecnología a través de una revisión teórica que finalmente permita obtener una línea de investigación en materia de usabilidad del e-gobierno bajo un sentido social, sin embargo, esa característica implícita de las variables hace que los creadores de políticas públicas omitan algunos elementos importantes en el desarrollo e implementación de herramientas de incorporación de la ciudadanía en la participación social.

\footnotetext{
CARRERA-MORA, Oscar Yahevh; OVANDO, María Catalina y VILLAFUERTE, Luis Fernando. La relación de la perspectiva de eficiencia del ciudadano con su comportamiento de uso de los servicios de e-gobierno municipal. Innovar, v. 29, n. 74, p. $133-146$. https://doi. org/10.15446/innovar.v29n74.82096. Acceso en: 03 feb. 2019.

4 EBBERS, Wolfgang; JANSENB, Marloes y VAN DEURSENA, Alexander. Impact of the digital divide on e-government: Expanding from channel choice to channel usage. Government Information Quarterly, v. 33, n. 4, p. 685-692, 2016. Doi: https://doi. org/10.1016/j.giq.2016.08.007.

5 LIANG Li; KUI Du; WEI Zhang y JI-YE Mao. Poverty alleviation through government-led e-commerce development in rural China: An activity theory perspective. Information System Journal, 2018. https://doi.org/10.1111/isj.12199.

6 UNITED NATIONS. Designing e-Government for the Poor. Economic and Social Commission for Asia and The Pacific Asian Development Bank Institute. 2005. Disponible en: https://www.adb.org/sites/default/files/publication/159381/adbi-e-gov-poor. pdf.

SANJO, Faniran y KAYODE, Olaniyan. e-Governance in Community-Based Poverty Reduction Strategies. Rights link, p. 144148, 2008. Disponible en: Acceso en:

8 LASAINE, Emma. E-Government: A Tool for Poverty Reduction?. Borgen Magazine, 2015. Disponible en: https://www.borgenmagazine.com/e-government-tool/. Acceso en:
} 
De lo anterior, se deduce hipotéticamente que los diferentes factores derivados de la pobreza tienen un efecto negativo en la inclusión de la ciudadanía a elementos como infraestructura, educación y habilidades digitales que van limitando la usabilidad de los servicios públicos brindados a través de plataformas web (e-gobierno). Por lo tanto, con este estudio se buscó desarrollar un diagrama causal de los factores identificados en esta revisión como: la falta de conocimiento de su existencia, analfabetismo digital, entre otros más identificados.

\section{Revisión de la literatura}

\subsection{E-gobierno en México}

La adopción de TIC por parte de la administración pública se ha derivado de diversos objetivos, de los cuales sobresalen la necesidad de propiciar eficiencia de los servicios públicos y la inclusión de todos los ciudadanos a éstos ${ }^{9}{ }^{10}$, por lo cual se han ido utilizando varios mecanismos para el logro de ellos, de entre los cuales esta el e-gobierno, se entiende por e-gobierno al uso de las TIC en la entrega de información vía Internet que proporciona la administración pública como medio para la entrega de servicios públicos ${ }^{11}{ }^{12}$, el e-gobierno comprende diferentes esferas de acción, como lo son: los servicios (e-servicios), la participación ciudadana (e-democracia), entre otros ${ }^{13}$.

La aplicación del e-gobierno y principalmente de los e-servicios ha implicado grandes transformaciones en los procesos gubernamentales en México que han requerido diseñar nuevas estructuras que permitan su desarrollo e implementación a través de acciones y/o políticas públicas impulsoras de los cambios orientados hacia una sociedad de la información y del conocimiento (SIC).

Su funcionamiento y éxito, depende de dos grandes dimensiones, bajo la perspectiva de diversos autores ${ }^{1415}$ para cumplir con los objetivos y ser exitosa la implementación y uso del e-gobierno, éste depende de dos esferas principales (ver Fig. 1).

\footnotetext{
CARRERA-MORA, Oscar Yahevh; OVANDO, María Catalina y VILLAFUERTE, Luis Fernando. La relación de la perspectiva de eficiencia del ciudadano con su comportamiento de uso de los servicios de e-gobierno municipal. Innovar, v. 29, n. 74, p. $133-146$. https://doi. org/10.15446/innovar.v29n74.82096. Acceso en: 03 feb. 2019.

10 CRUZ, Christian y ZAMUDIO, Ayesha. Municipios y gobierno abierto, más allá del gobierno electrónico. Opera, v. 21, p. 55-77, 2017. Disponible en: https://ssrn.com/abstract=3114492. Acceso en:

11 AS'AD, As'ad, KHAZAEI, Babak; AKHGAR, Babak y ALQATAWNA, Ja' far. importance of service integration in e-government implementations. In: INTERNATIONAL CONFERENCE ON INFORMATION AND COMMUNICATION, 7., 2016. Anales electrónicos [...]. [S.l.]: SYSTEMS (ICICS), 2016. p. 56-61. DOI: 10.1109/IACS.2016.7476086.

12 SANTA, Ricardo; MACDONALD, Jason y FERRER, Mario. The role of trust in e-Government effectiveness, operational effectiveness and user satisfaction: Lessons from Saudi Arabia in e-G2B. Government Information Quarterly, v. 36, n. 1, p. 39-50, 2019. Doi: https://doi.org/10.1016/j.giq.2018.10.007.

13 LUNA-REYES, Luis Felipe; GIL-GARCÍA, Ramón, y CELORIO, José Antonio. El mundo del e-gob local: evidencias de municipios digi- tales en el mundo. Ciudad de México: Infotec I+D+i, 2010.

${ }_{14}$ ALFARO, Rodrigo; BUSTOS, Guillermo; GONZÁLEZ; Alejandra y LOROÑO, Joseba. Introducción al gobierno electrónico: actores y dimensiones. Ediciones Universitarias de Valparaíso. Disponible en: http://www.euv.cl/archivos_pdf/gobierno-electronico.pdf. Acceso en: 02 feb. 2005.

15 MISHRA, Alok, y MISHRA, Deepti. E-Government - Exploring the Different Dimensions of Challenges, Implementation, and Success Factors. The Data Base for Advances in Information Systems, v. 42, n. 4, p. 23-37, 2011. Disponible en: https://www.researchgate.net/publication/220627546. Acceso en:
} 
Fig. 1. Actores y Dimensiones del e-gobierno.

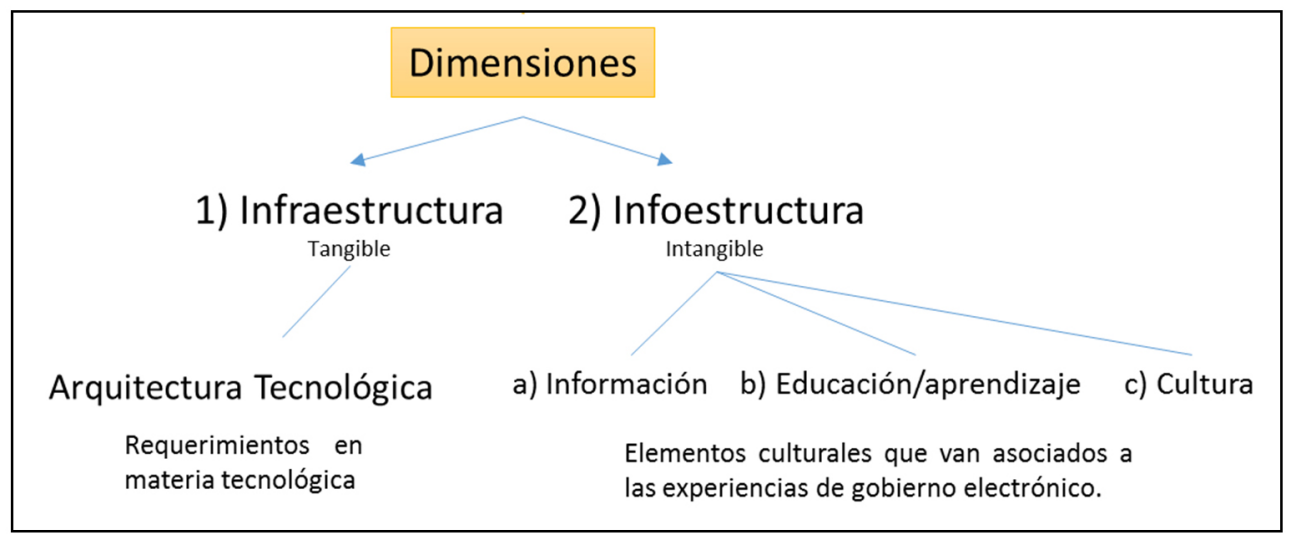

Fuente: elaboración propia.

La infraestructura, que en la era del internet se puede entender como una red suficientemente dimensionada, de fácil acceso, barata, abierta a ciudadanos y organizaciones e incluye todos los mecanismos de acceso como: redes, computadoras, internet, entre otros ${ }^{16}, \mathrm{y}$;

La infoestructura consiste en todo aquello que permite sacar rendimiento de la infraestructura ${ }^{17}$, un componente básico es el conocimiento derivado de la educación y de la alfabetización educativa, elementos que van asociados a elementos culturales, de información y de educación y aprendizaje.

En México, en los diferentes niveles de gobierno se han implementado este tipo de plataformas (e-servicios), impulsados por una estrategia de acceso como el programa México Conectado que fue parte de la Estrategia Digital Nacional (EDN) que entró en vigor en el año 2015, programa que buscó se otorgara el acceso gratuito a internet a toda la ciudadanía a través de infraestructura de internet móvil, fue colocada en las diversas plazas públicas de estados y municipios de México, así mismo, en materia de infoestructura, dotaron al país de centros de apoyo en materia de alfabetización digital que fueron puestos físicamente en las principales ciudades de cada estado de la republica mexicana.

Sin embargo, a pesar de ello, en materia de usabilidad de los e-servicios, aún hay mucha exclusión de los ciudadanos de las plataformas, pese a que se tiene definido un esquema y diversos modelos para el éxito del e-gobierno, por lo tanto, es importante conocer algunos de los factores que limitan a la población hacer uso de estos recursos.

En este sentido, la brecha digital juega un papel sumamente importante ${ }^{18}$, ya que genera exclusión, por lo cual, primero es importante explicar que derivado del surgimiento de la era de la informática y las telecomunicaciones, surgió un nuevo elemento de desigualdad denominado brecha digital ${ }^{19}$, la cual, se refería a la desigualdad entre aquellos que tenían o no tenían acceso físico a las TIC, pero hoy en día se tienen diferentes tipos de brechas digitales, en primer lugar, aquella que representa la brecha de acceso, en segundo lugar, la brecha de uso o también denominada brecha de segundo nivel ${ }^{20}$ y, por último, la brecha de apropiación.

\footnotetext{
16 SAURABH, Chandra. Infrastructure for e-Government Development Issues and Challenges in India. Dynamics of Public Administration, v. 35, n. 1, p. 129-149, 2018. Doi: 10.5958/0976-0733.2018.00010.X.

17 ABDUL, Aliza; HABIBAH, Noor y JANOM, Norjansalika. Understanding and Building the Definition for Infostructure in Disaster Management. Journal of Advanced Management Science, v. 4, n. 6, p. 511-514, 2016. Doi: 10.18178/joams.4.6.511-514.

18 MURITALA, Olaseni; ROWLE, Jennifer y JOHNSON, Frances. The multi-dimensional digital divide: Perspectives from an egovernment portal in Nigeria. Government Information Quarterly, v. 34, n. 2, p. 329-339, 2017. Doi: 10.1016/j.giq.2017.02.002.

19 GÓMEZ, Dulce Angelica; ALVARADO, Raúl Arturo; MARTÍNEZ, Marlen y DÍAZ DE LEÓN, Christrian. La brecha digital: una revisión conceptual y aportaciones metodológicas para su estudio en México. Entreciencias: Diálogos en la Sociedad del Conocimiento, v. 6, n. 16, p. 49-64, 2018. DOI: https://doi.org/10.22201/enesl.20078064e.2018.16.62611.

20 SEONG-JAE, Min. From the Digital Divide to the Democratic Divide: Internet Skills, Political Interest, and the Second-Level Digital Divide in Political Internet Use. Journal of Information Technology \& Politics, v.7, n. 1, p. 22-35, 2010. DOI: 10.1080/19331680903109402.
} 
Estas brechas propician limites, tanto de acceso a la infraestructura que afectan los canales de comunicación con los servicios públicos electrónicos, como al uso y la apropiación que se origina por otros factores que limitan la usabilidad, de hecho, según Ebbers, Jansenb y Van Deursena ${ }^{21}$ en los países desarrollados, ni el acceso, ni la situación socioeconómica son factores únicos de exclusión en el uso, también lo son las aptitudes digitales, por lo cual, el análisis del origen de estos factores es primordial para comprender como se generan estos límites, así mismo, la pobreza entendida como un elemento multidimensional es un elemento clave para comprender el origen de esos factores que conforman las brechas que como consecuencia generan exclusión de la ciudadanía en el uso de los e-servicios.

En este sentido, el uso del e-gobierno resulta una estrategia calificada como prioritaria por parte del gobierno de México para tratar de incluir a toda la ciudadanía en los procesos de dotación de servicios públicos, en esa tesitura, se incluye a los sectores sociales considerados dentro de los estratos de pobreza y pobreza multidimensional en el país. La estrategia gubernamental se alineo a los objetivos del desarrollo sostenible 2030, principalmente en el objetivo 9 que describe "industria, innovación e infraestructura" y el 10, "Reducción de la desigualdad", desde el sexenio del Presidente Enrique Peña Nieto (2012 - 2018) se estableció un programa denominado "Red compartida" que manejaba un esquema en donde el gobierno federal vendía a empresas privadas el servicio de banda ancha y, esta como una especie de intermediario, vendía Internet a bajo costo a los usuarios finales, al final del sexenio, se alcanzó a cubrir el 32.3\% de la población, sin embargo, significativamente, no tuvo un éxito en sus metas, ya que metodológicamente contabilizo por porcentaje de población y no por número de usuarios, por lo que se concentró el uso del internet en áreas urbanas, pero no se extendió a mayores zonas poblacionales con dispersión territorial, bajos índices económicos, es decir zonas marginadas.

Con la llegada del Presidente Andrés Manuel López Obrador 2018 - 2024) se implementó una nueva estrategia que se llama "Internet Para Todos", el cual cambia radicalmente la estrategia, la cual es; conectar gratis a la red inalámbrica a la población del país a través de el cableado de fibra óptica con que cuenta la Comisión Federal de Electricidad (CFE) de México a través de una nueva empresa "CFE Telecomunicaciones e Internet para Todos", siendo una inversión completamente pública. Esta fue creada el 2 de agosto del 2019, y su objetivo según el Diario Oficial de la Federación (DOF) es “prestar y proveer servicios de telecomunicaciones, sin fines de lucro, para garantizar el derecho de acceso a las tecnologías de la información y comunicación, incluido el de banda ancha e internet" ${ }^{22}$.

Sin embargo, este proyecto Impulsado por el Presidente López Obrador nació técnicamente muerto, ya que en este año y medio de gestión gubernamental no se ha mostrado un incremento significativo en el número de usuarios de telefonía con Internet, ni de conexión de en los hogares, ni en mejores servicios, según datos del Instituto Federal de telecomunicaciones (IFT) ${ }^{23}$ el número de celulares paso de 91 a 96 en el primer semestre de 2019, pero esta cifra nos dice mucho si hacemos notar que en la ciudad de México para este trimestre había 109 celulares por cada 100 habitantes, mientras que en Chiapas y Oaxaca cuentan con 60, es decir, al igual que el programa de Peña Nieto, este programa se concentra en poblaciones que ya cuentan con el servicio de Internet, más no en la población en condiciones de pobreza.

Y es que si bien en México ha crecido el número de usuarios de internet al pasar de 71.3 millones en 2017 a 74.3 millones en 2018, según datos de la Encuesta Nacional sobre Disponibilidad y Uso de Tecnologías de la Información en los Hogares (ENDUTIH) ${ }^{24}$, las diferencias son claras, el uso del internet es un fenómeno

\footnotetext{
21 EBBERS, Wolfgang; JANSENB, Marloes y VAN DEURSENA, Alexander. Impact of the digital divide on e-government: Expanding from channel choice to channel usage. Government Information Quarterly, v. 33, n. 4, p. 685-692, 2016. Doi: https://doi. org/10.1016/j.giq.2016.08.007.

22 MÉXICO. Diario Oficial de la Federación. 2019. Disponible en: https://www.dof.gob.mx. Acceso en:

23 MÉXICO. Instituto Federal de Telecomunicaciones. 2019. Disponible en: http://www.ift.org.mx/pagina-de-inicio/informesestadisticos-trimestrales. Acceso en:

24 MÉXICO. Encuesta Nacional sobre Disponibilidad y Uso de Tecnologías de la Información en los Hogares (ENDUTIH). 2019. Disponible en: https://www.inegi.org.mx/programas/dutih/2019/. Acceso en:
} 
urbano, puesto que el 73.1\% del total de la población urbana son usuarios de este servicio, en cambio, sólo 40.6\% de la población conectada está en zonas rurales. Según la encuesta hay 29.9 millones que no tienen acceso a este servicio, y de ellos, 4 de cada 10 dicen que es por falta de recursos para costearlo, mientras que $2 \%$ no lo usa por falta de cobertura. Esto demuestra como la brecha digital tiene una relación directa con la brecha económica.

Entonces el programa actual parte de implementar la conectividad del internet en áreas donde ya existía, es decir, ofreciendo servicios a los que ya lo tenían y sólo a mediano y largo plazo, según la IFT en promedio en 10 años se calcula que se pueda llegar a zonas sin conectividad. Para que funcione la estrategia y se pueda llegar a las zonas rurales en 2024, se deberá desplegar infraestructura, pero también atraer a concesionarios o comercializadores autorizados por el IFT con el fin de reventa de la capacidad y servicios de la red a la población. Sólo así podría conseguirse que el proyecto sea exitoso. La idea del proyecto es alcanzar las 47 mil localidades donde vive 5\% de la población en el país a donde no ha llegado ninguna empresa de telecomunicaciones. Sin embargo, hacerlo con fibra óptica resulta poco viable, pues este tipo de usos es muy útil cuando se aplica en contextos en que la densidad poblacional es muy alta, pero cuando, por ejemplo, según el proyecto, se va a llegar a poblaciones con 50 habitantes, el costo del uso de la fibra óptica es muy alto, en donde funcionarían mejor otro tipo de tecnologías, como por ejemplo la satelital.

Así, el uso de estos programas tendría que pensarse muy bien en cómo implementarlos, de tal forma que las políticas públicas creadas para esta discusión sean basadas no solo en elementos técnicos, sino también en la consideración de diversos factores que son generados por una condición de pobreza, con lo cual estos individuos solo se abocaran a la utilización de las herramientas del e- gobierno.

Una exhaustiva revisión de la literatura ha permitido identificar que los estudios de e-gobierno en los últimos años centran su atención principalmente en dos aspectos (ver Tabla 1), primero, los factores y elementos requeridos y derivados de la implementación, por lo cual, cuando se realizan en conjunto con la variable de pobreza, teniendo como objeto de estudio las limitantes para su implementación como lo es: la brecha digital, que genera exclusión de los ciudadanos, los desafios y oportunidades que estos representan, así mismo, se observa la implementación del e-gobierno como un elemento que debe buscar la erradicación de la pobreza, el segundo aspecto o dimensión de estudio, es el uso o usabilidad, una vez que el e-gobierno esta implementado, las investigaciones versan sobre indagar todos aquellos factores que favorecen tras la usabilidad del mismo, por lo tanto, el objeto de estudio principal ha sido verificar el impacto de estos mecanismos en la pobreza, así como, indagar como el e-gobierno favorece la reducción de la pobreza.

Tabla 1. Dimensiones de estudio del e-gobierno con objeto de estudio la pobreza

\begin{tabular}{|c|c|c|}
\hline Dimensiones de Estudio & Objeto de estudio & Autores \\
\hline $\begin{array}{c}\text { a) Implementación del } \\
\text { E-gobierno }\end{array}$ & $\begin{array}{l}\text { - Politicas públicas para erradicar } \\
\text { la pobreza. } \\
\text { - Desafios y Oportunidades. } \\
\text { - Brecha digital }\end{array}$ & $\begin{array}{l}\text { EBBERS, Wolfgang; JANSENB, Marloes y VAN } \\
\text { DEURSENA, Alexander. } 2016 \\
\text { - MISHRA, Alok, y MISHRA, Deepti. 2011. } \\
\text { - MUKHOPADHYAY, Sandip; BOUWMAN, Harry } \\
\text { y PRASAD, Mahadeo. } 2019 \text {. } \\
\text { - MURITALA, Olaseni; ROWLE, Jennifer y } \\
\text { JOHNSON, Frances. 2017. } \\
\text { - SAURABH, Chandra. } 2018 \\
\end{array}$ \\
\hline $\begin{array}{l}\text { b) Uso del } \\
\text { E-gobierno }\end{array}$ & $\begin{array}{l}\text { Impacto en la pobreza. } \\
\text { - Reducción de la pobreza. }\end{array}$ & $\begin{array}{l}\text { - LASAINE, Emma. } 2015 . \\
\text { - LIANG Li; KUI Du; WEI Zhang y JI-YE Mao. } \\
\text { MUTULA, Stephen. } 2013 . \\
\text { - SANJO, Faniran y KAYODE, Olaniyan. } 2008 . \\
\text { - SAXENA, K.B.C., y CHAHUAN, Kalpana. } 2013 . \\
\text { - SPENCE, Randy y SMITH, Matthew. } 2010 .\end{array}$ \\
\hline
\end{tabular}

Fuente: elaboración propia.

Si embargo, con este estudio no solo se trata de señalar a la pobreza como un fenómeno de exclusión que es restrictivo del uso de las TIC y decidirse por visualizar otros escenarios. La propuesta de este estudio radica en abordar como estas condicionantes definidas como variables o factores que surgen de una condición 
de pobreza tienen una causalidad específica sobre la usabilidad del e-gobierno que necesita hacerse explicita, representado a través de un diagrama causal, acción que pudiera permitir a los hacedores de políticas públicas tener una noción más clara de todos los factores y variables que pueden surgir como una limitante en la usabilidad de las TIC, posterior a la implementación de estos mecanismos.

\subsection{La pobreza en México}

El entendimiento de la pobreza es fundamental, ya que, gran parte de la población en México tiene ingresos en el límite o por debajo de la línea de bienestar económico, lo que lleva a la ciudadanía en situación de pobreza a cubrir necesidades básicas generando diversas barreras de exclusión ${ }^{25}$. La pobreza es un problema que no se ha podido erradicar durante décadas a nivel mundial, por lo cual en este análisis no se buscó definir alternativas para combatirla, si no comprender los factores que emanan de ella para favorecer al diseño de alternativas que permitan a la ciudadanía en esa situación acceder a los e-servicios, pudiendo ver al e-gobierno como un elemento potencial para acercar la administración pública a las personas en condición de pobreza ${ }^{26}$.

Desafortunadamente, cuando se habla de pobreza el pensamiento nos dirige inmediatamente a una situación o condición en la que una persona no puede acceder a una serie de recursos, principalmente los económicos, pero también nos debería dirigir el pensamiento hacía alternativas y estrategias para mitigarla ${ }^{27}$, sin embargo, es claro que hay muchas formas de definirla, por ello es importante analizar las diferentes dimensiones para su abordaje, por lo tanto, en este análisis la concepción sobre pobreza ${ }^{28}$ concebida en los objetivos de desarrollo sostenible "Fin de la pobreza" representa una perspectiva adecuada para comprenderla, ya que explica que la pobreza va más allá de la falta de ingresos y recursos para garantizar unos medios de vida sostenibles, considerando como pobreza también a la falta de servicios básicos, la discriminación, la exclusión social y la falta de participación en la adopción de decisiones públicas.

No obstante, los paradigmas de la pobreza según los ingresos son fundamentales para comprender de inicio algunos factores derivados de carencias y necesidades básicas del ser humano que llevan a una comprensión e interpretación del medio para poder establecer como hay factores de la pobreza que limitan la participación de la ciudadanía en diversas áreas del consenso público y de servicios públicos como el e-gobierno.

En México el Consejo Nacional de Evaluación de la Política de Desarrollo Nacional (CONEVAL), es el encargado de establecer los patrones para la medición de pobreza económica, definiendo para su análisis dos líneas de ingreso: primero, la Línea de Pobreza Extrema por Ingresos, que equivale al valor de la canasta alimentaria por persona al mes; y segundo, la Línea de Pobreza por Ingresos, que equivale al valor total de la canasta alimentaria y de la canasta no alimentaria por persona al mes.

En ese sentido, en México según datos del CONEVAL ${ }^{29}$, el $41.9 \%$ de la población que equivale a 52.4 millones de personas se encuentran en situación de pobreza, el 7.4\% que equivale a 9.3 millones de personas esta en situación de pobreza extrema, así mismo, un $6.9 \%$ es vulnerable por ingresos y el $29.3 \%$ que equivale a 36.7 millones de personas es una población vulnerable por carencias sociales, lo cual deja de manifiesto que el 48.8\% de la población tiene un ingreso inferior a la línea de pobreza por ingresos, en consecuencia, prác-

25 UNITED NATIONS. Objetivos de desarrollo sostenible. 2019. Disponible en: https://www.un.org/sustainabledevelopment/ es/poverty/. Acceso en:

26 LIANG Li; KUI Du; WEI Zhang y JI-YE Mao. Poverty alleviation through government-led e-commerce development in rural China: An activity theory perspective. Information System Journal. https://doi.org/10.1111/isj.12199.

27 SANJO, Faniran y KAYODE, Olaniyan. e-Governance in Community-Based Poverty Reduction Strategies. Rights link, p, 144148, 2008. Disponible en: Acceso en:

${ }_{28}$ UNITED NATIONS. Objetivos de desarrollo sostenible. 2019. Disponible en: https://www.un.org/sustainabledevelopment/ es/poverty/. Acceso en:

29 MÉXICO. Consejo Nacional de Evaluación de la Política de Desarrollo Social. Pobreza en México, medición de la pobreza. Disponible en: http://webdrp.coneval.org.mx/Medicion/MP/Paginas/Pobreza-2018.aspx. Acceso en: 03 feb. 2018. 
ticamente en la mitad de la población los factores definidos en esta investigación tienen algún efecto sobre la ciudadanía. "El hecho de que en 2016 el 43,6\% de la población viviera en pobreza, describe un panorama poco alentador y una gran fragilidad del tejido social del país"30.

Sin embargo, "puede haber dos países con igual tasa de pobreza según ingresos, pero desigual cobertura en los servicios sociales" ${ }^{31}$, por lo cual es importante dimensionar la pobreza desde diferentes ángulos y perspectivas para un análisis más profundo.

\subsubsection{Dimensiones de la pobreza}

La pobreza no es un elemento unidimensional, por ello para su estudio es necesario comprender y establecer las diferentes dimensiones que abarca, la definición de indicadores y umbrales en dimensiones distintas al ingreso entregaría una visión más completa respecto del bienestar de la población considerando dos dimensiones ${ }^{18} 32$ : primero, bienestar económico, representado por el indicador "ingreso corriente per cápita del hogar"; y segundo, derechos sociales, que abarca seis indicadores a) Rezago educativo, b) Acceso a los servicios de salud, c) Acceso a la seguridad social, d) Calidad y espacios de la vivienda, e) Acceso a los servicios básicos en la vivienda y f) Acceso a la alimentación.

Por otra parte, esta el Índice de Pobreza Humana (IPH) definido por el Programa de las Naciones Unidas para el Desarrollo que abarca tres dimensiones básicas, vida larga y saludable, educación y nivel de vida digna. Y, por último, tenemos a la Oxford Poverty \& Human Development Iniciative (OPHI) ${ }^{33}$ que destaca cinco dimensiones, basadas en el método Alkire y Foster para la medición multidimensional de pobreza, mismo que ha sido utilizado por los formuladores de políticas en países de todo el mundo para crear medidas nacionales de pobreza y bienestar, las dimensiones son a) Condiciones educativas, b) Condiciones de la niñez y la juventud, c) El trabajo, d) La salud y e) Las condiciones de la vivienda.

Por lo cual, dadas todas esas dimensiones y variables que se pueden presentar, la condición de pobreza puede derivar en factores de exclusión de la ciudadanía en los e-servicios, mismos que es necesario comprenderlos para generar alternativas que permitan reducir la problemática de exclusión.

En cuanto a los indicadores de la pobreza multidimensional, también hay diferentes perspectivas, sin embargo, para esta investigación se tomaron dos en particular, primero, la del Oxford Poverty \& Human Development Iniciative (OPHI) organización que plantea 10 indicadores: nutrición, mortalidad infantil, años de escolaridad, asistencia escolar, electricidad, saneamiento, bienes, alimentación, agua potable y vivienda, y segundo, la del CONEVAL ${ }^{34}$ que plantea la medición a partir de ocho indicadores, en donde, "considera el ingreso y seis dimensiones en el enfoque de los derechos sociales. Esta perspectiva se complementa con la incorporación de la cohesión social, para reconocer la importancia de factores contextuales y relacionales".

El interés particular de buscar la inclusión de las personas en esta condición, se debe a que la prestación

30 VALERA, Rogelio y HERnÁNDEZ, Juan Manuel. Pobreza multidimensional y mercado laboral en México. Cuadernos de Economía, v. 39, n. 79, p. 144, 2019. DOI: 10.15446/cuad.econ.v39n79.71201.

31 LARRAÑAGA, Osvaldo. La medición de la pobreza en dimensiones distintas al ingreso. CEPAL: Serie Estudios estadísticos y prospectivos, n. 58, p. 11, 2007. Disponible en: https://repositorio.cepal.org/bitstream/handle/11362/4760/S0700697_ es.pdf? sequence $=1 \&$ is Allowed $=\mathrm{y}$. Acceso en:

32 SCOTT, John; GUTIÉRREZ, Aida, y CHÁVEZ, Carolina. Medición multidimensional de la pobreza rural en México acceso efectivo y nuevas dimensiones. Instituto de Estudios Peruanos. 2019. Disponible en: http://repositorio.iep.org.pe/handle/IEP/9. Acceso en:

33 OXFORD POVERTY AND HUMAN DEVELOPMENT INITIATIVE. Global multidimensional poverty index 2019: illuminating inequalities. United Nations Development Programme and Oxford Poverty and Human Development Initiative. 2019. Disponible en: http://hdr.undp.org/sites/default/files/mpi_2019_publication.pdf. Acceso en:

34 MÉXICO. Consejo Nacional de Evaluación de la Política de Desarrollo Social. Medición multidimensional de la pobreza en México: un enfoque de bienestar económico y de derechos sociales. Diponible em: https://www.coneval.org.mx/InformesPublicaciones/ FolletosInstitucionales/Documents/Medicion-multidimensional-de-la-pobreza-en-Mexico.pdf. P. 2. Acceso en: 05 feb. 2019. 
eficiente de servicios gubernamentales a los pobres, se enfrenta a muchos desafíos ${ }^{35}$, debido a que, la situación de pobreza tiene una profunda relación con los límites de la ciudadanía con el uso del e-gobierno en cuanto a las diferentes dimensiones de infraestructura como de infoestructura y, lamentablemente, en los países en desarrollo como México muchas familias marginadas no tienen acceso a la información básica que necesitan para tomar decisiones críticas y aprovechar los programas de bienestar social y hacer uso de los e-servicios tan necesarios ${ }^{36}$.

Así mismo, autores ${ }^{37}$ manifiestan que el aumento del acceso a la asistencia federal basada en TIC ofrece a los ciudadanos empobrecidos un medio para mejorar su calidad de vida, reduciendo la incidencia general de la pobreza, ya que un servicio basado en TIC permite reducir los costos de traslado para el ciudadano y los relacionados con la prestación de servicios, liberando así, recursos para ofrecer un mayor volumen de asistencia por parte del gobierno, así mismo, al facilitar el acceso, el e-gobierno pone los servicios gubernamentales a disposición de un mayor número de personas de lo que antes era posible.

Por lo tanto, hay diferentes elementos o indicadores que afectan la usabilidad o la intención de uso de la ciudadanía, mismos que se derivan de una situación de pobreza, ya que las personas en situación de pobreza toman todas sus decisiones en un contexto de escasez, en este contexto la escasez se traduce en términos de la infraestructura y la infoestructura definidas en párrafos anteriores.

\section{Metodología}

La presente es una investigación de tipo descriptivo - explicativo y se fundamentó en lo siguiente:

\subsection{Dimensiones e indicadores de la pobreza en México}

Para poder establecer un marco de trabajo (ver Fig. 1) que permita definir las variables que tienen un efecto sobre la usabilidad del e-gobierno, se tomó como dimensión de estudio de la pobreza para esta investigación a la clasificación descrita la Oxford Poverty \& Human Development Iniciative ${ }^{38}$ que destaca cinco dimensiones y para los indicadores han sido abstraídos de las variables definidas por el Índice de Desarrollo Humano, ${ }^{39}$ el OPHI ${ }^{40}$ y el CONEVAL ${ }^{41}$, permitiendo plantear los diferentes indicadores que se agrupan en cada una de las dimensiones del e-gobierno. De esta forma, es como se establecen los indicadores surgidos de la pobreza que afectan la usabilidad del e-gobierno (ver Fig. 2).

\footnotetext{
35 MUKHOPADHYAY, Sandip; BOUWMAN, Harry y PRASAD, Mahadeo. An open platform centric approach for scalable government service delivery to the poor: the Aadhaar case. Government Information Quarterly v. 36, n. 3, p. 437-448, 2019. Doi: 10.1016/j. giq.2019.05.001.

36 SAXENA, K.B.C., y CHAHUAN, Kalpana. E-Governance for Rural Poor: Issues \& Challenges. FIIB Business Review, v. 2, n. 4, 2013. Doi: $10.1177 / 2455265820130402$.

37 SPENCE, Randy y SMITH, Matthew. ICT, Development, and Poverty Reduction: Five Emerging Stories. Information Technologies \& International Development, v. 6, Special Edition, p. 11-17, 2010.

38 OXFORD POVERTY AND HUMAN DEVELOPMENT INITIATIVE. Global multidimensional poverty index 2019: illuminating inequalities. United Nations Development Programme and Oxford Poverty and Human Development Initiative. 2019. Disponible en: http://hdr.undp.org/sites/default/files/mpi_2019_publication.pdf. Acceso en:

39 Índice de Desarrollo Humano. Índices e indicadores de desarrollo humano. Disponible en: http://hdr.undp.org/sites/default/ files/2018_human_development_statistical_update_es.pdf. Acceso en:

40 OXFORD POVERTY AND HUMAN DEVELOPMENT INITIATIVE. Global multidimensional poverty index 2019: illuminating inequalities. United Nations Development Programme and Oxford Poverty and Human Development Initiative. 2019. Disponible en: http://hdr.undp.org/sites/default/files/mpi_2019_publication.pdf. Acceso en:

41 MÉXICO. Consejo Nacional de Evaluación de la Política de Desarrollo Social. Medición multidimensional de la pobreza en México: un enfoque de bienestar económico y de derechos sociales. https://www.coneval.org.mx/InformesPublicaciones/FolletosInstitucionales/Documents/Medicion-multidimensional-de-la-pobreza-en-Mexico.pdf. Acceso en: 05 feb. 2019.
} 
Fig. 2. Dimensiones e indicadores de la pobreza en México

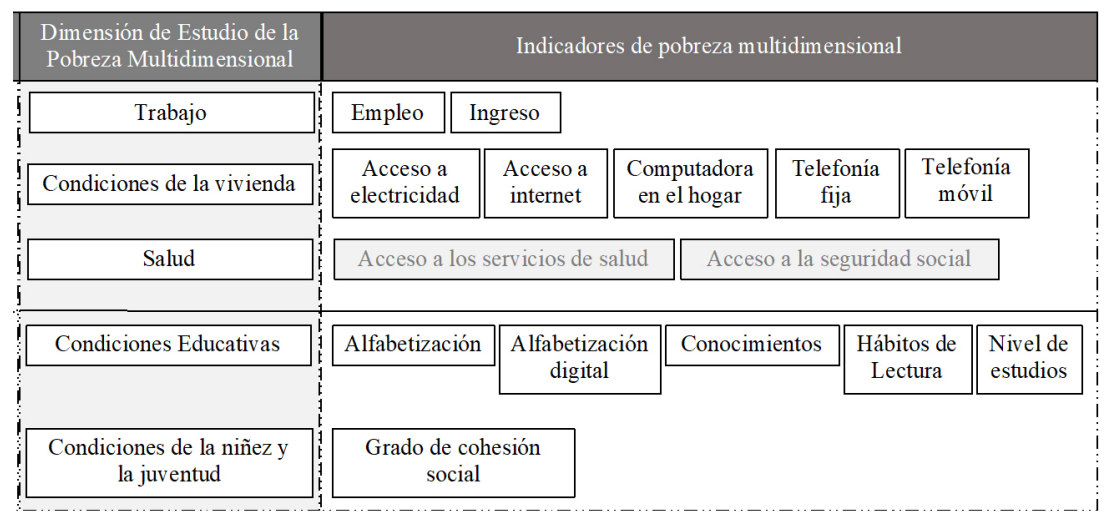

Fuente: elaboración propia.

\subsection{Dimensiones de estudio del e-gobierno}

Las causas que acentúan la usabilidad del e-gobierno, derivadas de la pobreza, se analizan a partir de una noción del pensamiento sistémico, como lo es la dinámica de sistemas; metodología que permite comprender la operación de sistemas complejos, como los sociales, por medio de la modelación y la simulación por computadora, permitiendo analizar cómo las interrelaciones de un sistema afectan el comportamiento y sus cambios en el tiempo ${ }^{42}{ }^{43}$, ver Fig. 3.

Por lo que, la investigación presenta únicamente un diagrama causal basado en la dinámica de sistemas que permitan identificar de manera cualitativa la inferencia de los indicadores de la pobreza en las dimensiones de éxito del e-gobierno.

La dinámica de sistemas, es una metodología utilizada en escasas investigaciones de e-gobierno en México, por su origen cualitativo, no obstante, existen avances en el tema de diferentes autores ${ }^{45}$ quienes utilizan la simulación dinámica para comprender el e-gobierno bajo un caso de estudio de los portales web del sistema e-México; así mismo "una teoría de colaboración basada en la retroalimentación, que contribuya a la comprensión de la interacción entre desarrollo de confianza y colaboración en proyectos interorganizacionales dentro del sector público" a través del e-gobierno ${ }^{46}$.

42 ROBLEDO, Jorge y FERNANDO, Yony. Estudio de un proceso de innovación utilizando la dinámica de sistemas. Cuadernos de Administración, v. 21, n. 35, p. 127-159, 2008. Disponible en: http://www.scielo.org.co/pdf/cadm/v21n35/v21n35a06.pdf. Acceso en:

43 QUINTERO, Danny y LÓPEZ, Sandra. Análisis estructural: un apoyo para el modelado con dinámica de sistemas. Revista Avances en Sistemas e Informática, v. 7, n. 3, 2010. Disponible en: http://www.bdigital.unal.edu.co/28807/1/26663-93574-1-PB.pdf. Acceso en:

44 MORLAN, Iñaki. Modelo de dinámica de sistemas para la implantación de tecnologías de la información en la gestión estratégica universitaria. Tesis - Universidad del País Vasco / Euskal Herriko Unibertsitatea, 2010.

45 GIL-GARCÍA, Ramón y LUNA, Luis Felipe. Teoría institucional y simulación dinámica para una mejor comprensión del gobierno electrónico: el caso de los portales web del sistema e-México. Gestión y Política Pública, México, CIDE, n. 247, 2010.

46 LUNA-REYES, Luis Felipe. Trust and collaboration in interorganizational information technology projects in the public sector. Gestión y Política Pública, Volumen Temático sobre Gobierno Electrónico, p. 171-210, 2013. p. 172. Disponible en: http://www. gestionypoliticapublica.cide.edu/VolTem_Gobierno_Electronico/LunaReyes_2013_eng.pdf. Acceso en: 
Fig. 3. Dimensiones de estudio del e-gobierno

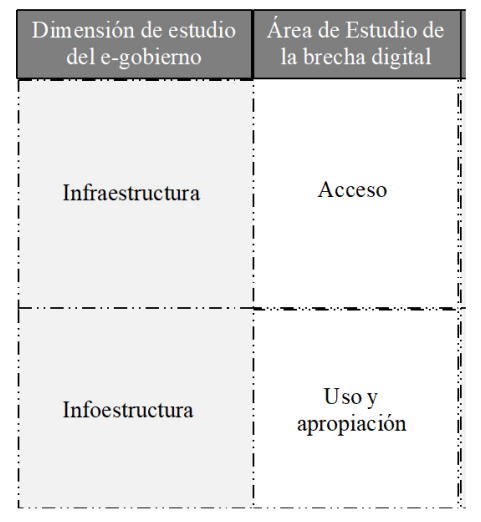

Fuente: elaboración propia.

\section{Resultados}

\subsection{Indicadores de la Pobreza que afectan al e-gobierno}

Las dimensiones de estudio de la pobreza multidimensional para este análisis han sido clasificadas de acuerdo con su efecto en las áreas de estudio de la brecha digital y estas a su vez, relacionadas con las dimensiones de estudio del e-gobierno, con la finalidad de tener claro la relación de los indicadores de la pobreza que tienen un efecto en la usabilidad del e-gobierno (ver Fig. 4), lo cual lleva a la construcción de un diagrama causal.

Fig. 4. Indicadores de la pobreza que afectan al e-gobierno

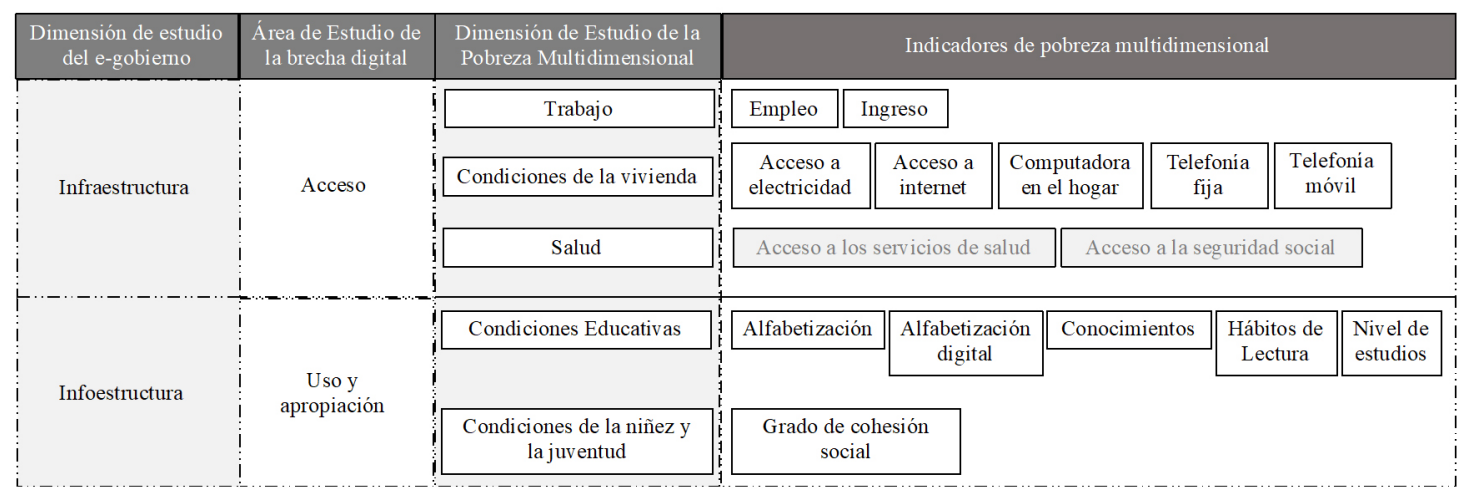

Fuente: elaboración propia.

Por lo tanto, la construcción de este marco de trabajo, permite visualizar a través de categorías la relación de las cinco dimensiones y de sus indicadores, cabe menciona que la dimensión de la pobreza multidimensional de salud, no es utilizada en este estudio, ya que no tiene una relación directa ni con la brecha digital, ni con el e-gobierno, la salud tiene definitivamente un efecto en la capacidad económica de las personas y otras variables más, pero no es una relación directa, motivo por el cual se excluye del diagrama causal. 


\subsection{Causalidad de la pobreza en la usabilidad del e-gobierno}

La comprensión de la causalidad de cada factor de la pobreza con el uso del e-gobierno se puede comprender a través del diagrama causal — diagrama de Forrester- (ver Fig. 5), en el cual, los indicadores de la pobreza se convierten en variables que afectan a las dimensiones del e-gobierno, por ejemplo, se puede observar que a mayor $(+)$ ingreso de los ciudadanos, existe un mayor acceso a la educación propiciando mejores condiciones educativa, pero también mejores condiciones de vivienda, afectando tanto a la dimensión de infraestructura como de infoestructura. Por lo tanto, si existe mayor pobreza (moderada y extrema), existirá una mayor brecha digital, a causa de las necesidades primarias de bienestar que contraen los ciudadanos al tener ingresos inferiores a la línea de bienestar ${ }^{47}$.

Fig. 5. Diagrama causal de factores que afectan la usabilidad del e-gobierno.

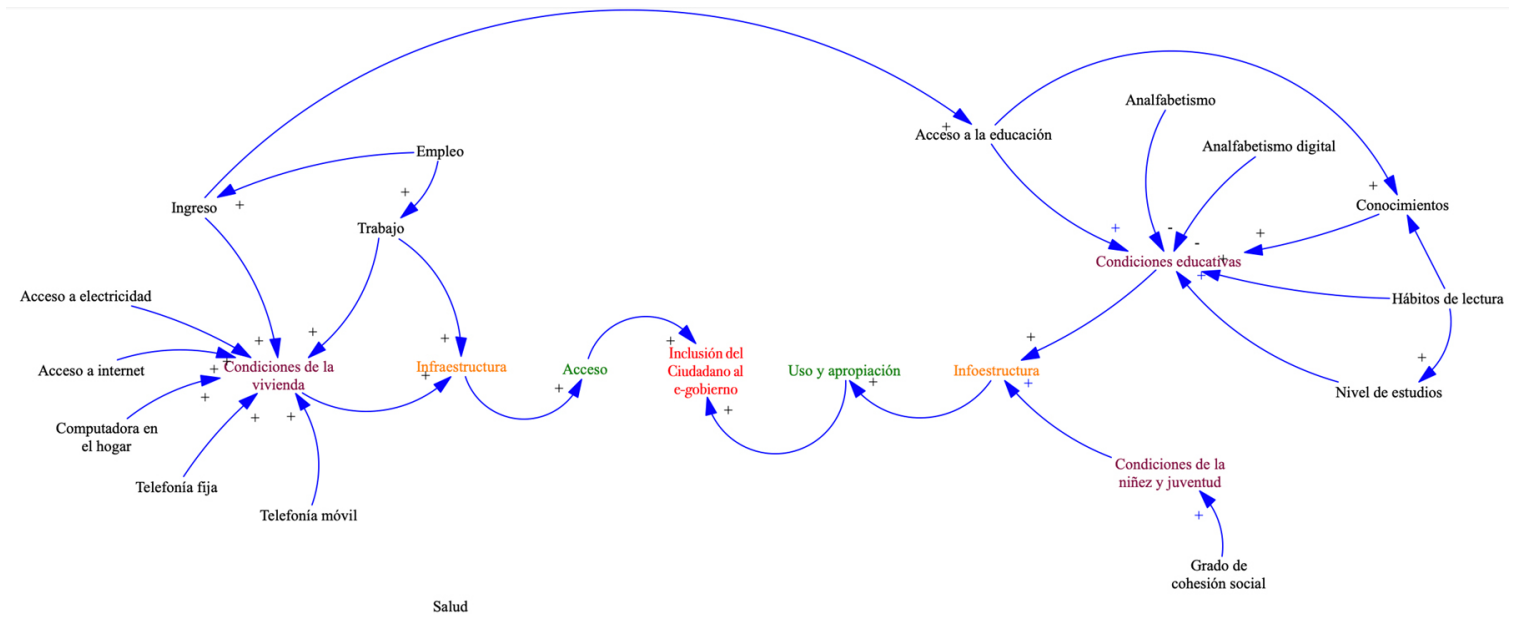

Fuente: elaboración propia.

Por otra parte, tenemos que, si existe mayor analfabetismo, las condiciones educativas tienen un decremento (-), impidiendo mejorar la capacidad de infoestructura de la ciudadanía, lo cual tiene un claro efecto en el uso y apropiación de la tecnología. De tal modo que, con este diagrama causal, es posible advertir a los diseñadores de las políticas públicas las bases necesarias para el mejor funcionamiento y éxito en la implementación de mecanismos de e-gobierno.

En estudios previos realizados por otros autores en cuanto a brecha digital ${ }^{48} \mathrm{y}$ de las dimensiones de la pobreza $^{49}{ }^{50}$, los ingresos y el rezago educativo (entendido como analfabetismo), son variables que resaltan a la hora de visualizar una posible disminución de la pobreza y la brecha digital, debido a que son elementos que limitan las posibilidades de resarcir ambas problemáticas, por tal motivo, se infiere que estas variables también son causales del aumento en la exclusión social en el e-gobierno. Por lo cual, a través de este contexto, y los ejes teóricos se puede percibir una relación de las variables a) bajos ingresos y b) analfabetismo, con las dimensiones de la pobreza, con la imposibilidad de disminuir la brecha digital y con la falta de inclusión de la ciudadanía al e-gobierno.

\footnotetext{
47 MÉXICO. Consejo Nacional de Evaluación de la Política de Desarrollo Social. Medición de la pobreza en México y en las entidades federativas. Disponible en: http://www.coneval.gob.mx/Medicion/Documents/Pobreza\%202014_CONEVAL_web.pdf. Acceso en: 04 feb. 2014.

48 SANDOVAL, Rodrigo. Explorando la brecha digital en México: Diagnóstico del proyecto e-México en el Estado de México. Espacios Públicos, p. 292-306, 2006. Disponible en: http://www.redalyc.org/articulo.oa?id=67601717. Acceso en:

${ }_{49}$ MÉXICO. Consejo Nacional de Evaluación de la Política de Desarrollo Social. Medición de la pobreza en México y en las entidades federativas. Disponible en: http://www.coneval.gob.mx/Medicion/Documents/Pobreza\%202014_CONEVAL_web.pdf. Acceso en: 04 feb. 2014.

50 MÉXICO. Instituto Nacional de Geografía y Estadistica. Disponible en: https://www.inegi.org.mx/datos/2018. Acceso en:
} 


\section{Conclusiones}

En esta investigación se parte de establecer un análisis de la correlación entre el e - gobierno, la pobreza y su utilidad en los procesos de gestión pública en el país, aunque la correlación podría ser aparentemente muy simple, la intención de este artículo es el hacer un primer acercamiento al tema para que sirva de punto de partida para futuras investigaciones sobre el tema.

Las líneas en que se desarrolla el artículo son tres:

A) Empezar a desarrollar una visión metodológica desde el enfoque sistémico para el análisis del ciclo de las políticas públicas, el cual nos permita analizar los escenarios previos al diseño y/o ejecución de la política pública para favorecer la usabilidad del e-gobierno, permitiendo con estos escenarios proporcionar datos cercanos a la realidad del éxito o fracaso de la decisión a tomar, teniendo como efecto de ello, la disminución en los costos de transacción, una toma de decisiones más asertiva y un panorama futuro de la inclusión social en el e-gobierno a generar.

B) Desarrollar una conceptualización teórica acerca del e-gobierno y su utilización en México, el cual, el desarrollo de los posicionamientos académicos está apenas vinculando el contexto en el uso de las plataformas digitales utilizadas en el servicio público, por lo que se usa el enfoque sistémico para hacer esa vinculación de las herramientas tecnológicas con el sentido social del uso de estas herramientas, estableciendo un marco analítico multidimensional y complejo.

C) A partir del estudio multidimensional mencionado líneas arriba, se incorpora el análisis de la pobreza como un factor del éxito o no del gobierno electrónico, tratando de dar propuestas para poder revertir esta situación, ya que el uso de las herramientas del e - gobierno pueden ser una estrategia muy positiva para tratar de establecer políticas públicas exitosas en un entorno social con alta marginación como es México, por lo que el poder presentar propuestas que lograran articular el uso del e - gobierno con la toma de decisiones más allá del uso que se le dé en relación al poder adquisitivo o el nivel educativo de sectores de la sociedad.

Por esta razón se inicia esta línea de investigación, la cual consideramos que puede ser una alternativa muy exitosa para empezar a realizar políticas públicas exitosas, las cuales, a partir de un enfoque de gobernanza, con un presupuesto participativo, con transparencia y rendición de cuentas, puede ser una herramienta que permita sacar de la pobreza y generar buenas políticas en el país.

Finalmente, es importante señalar que en este momento la investigación presentó variables generadas de una forma cualitativa, que son importantes para un conocimiento general de aquellos elementos originados por una condición de pobreza que tienen un efecto tanto positivo como negativo en la usabilidad del e-gobierno, sin embargo, esta línea de investigación permitirá en un siguiente ensayo ponderar cada una de las variables y a través de la misma metodología de dinámica de sistemas, se intentara presentar en un futuro escenarios simulados tanto optimistas como pesimistas que faciliten realizar predicciones de los resultados obtenidos al implementar políticas publicas que ayudan a intentar favorecer en las variables de la pobreza identificadas en este estudio como factores limitantes de la usabilidad del e-gobierno, pudiendo con ello trazar estrategias claras.

\section{Fuentes de información}

ABDUL, Aliza; HABIBAH, Noor y JANOM, Norjansalika. Understanding and building the definition for infostructure in disaster management. Journal of Advanced Management Science, v. 4, n. 6, p. 511-514, 2016. Doi: 10.18178/joams.4.6.511-514. 
ALFARO, Rodrigo; BUSTOS, Guillermo; GONZÁLEZ; Alejandra y LOROÑO, Joseba. Introducción al gobierno electrónico: actores y dimensiones. Ediciones Universitarias de Valparaíso. Disponible en: http://www. euv.cl/archivos_pdf/gobierno-electronico.pdf. Acceso en: 02 feb. 2005

AS'AD, As'ad, KHAZAEI, Babak; AKHGAR, Babak y ALQATAWNA, Ja'far. importance of service integration in e-government implementations. In: INTERNATIONAL CONFERENCE ON INFORMATION AND COMMUNICATION, 7., 2016. Anales electrónicos [...]. [S.l..]: SYSTEMS (ICICS), 2016. p. 56-61. DOI: 10.1109/IACS.2016.7476086.

CARRERA-MORA, Oscar Yahevh; OVANDO, María Catalina y VILLAFUERTE, Luis Fernando. La relación de la perspectiva de eficiencia del ciudadano con su comportamiento de uso de los servicios de egobierno municipal. Innovar, v. 29, n.74, p. 133-146, 2019. Disponible en: https://doi.org/10.15446/innovar. v29n74.82096. Acceso en: 03 feb. 2019.

CRUZ, Christian y ZAMUDIO, Ayesha. Municipios y gobierno abierto, más allá del gobierno electrónico. Opera, v. 21, p. 55-77, 2017. Disponible en: https://ssrn.com/abstract=3114492. Acceso en:

EBBERS, Wolfgang; JANSENB, Marloes y VAN DEURSENA, Alexander. Impact of the digital divide on e-government: expanding from channel choice to channel usage. Government Information Quarterly, v. 33, n. 4, p. 685-692, 2016. Doi: https://doi.org/10.1016/j.giq.2016.08.007.

GIL-GARCÍA, Ramón y LUNA, Luis Felipe. Teoría institucional y simulación dinámica para una mejor comprensión del gobierno electrónico: el caso de los portales web del sistema e-México. Gestión y Politica Pública, México, CIDE, n. 247, 2010.

GÓMEZ, Dulce Angélica; ALVARADO, Raúl Arturo; MARTÍNEZ, Marlen y DÍAZ DE LEÓN, Christian. La brecha digital: una revisión conceptual y aportaciones metodológicas para su estudio en México. Entreciencias: Diálogos en la Sociedad del Conocimiento, v. 6, n. 16, p. 49-64, 2018. DOI: https://doi. org/10.22201/enesl.20078064e.2018.16.62611.

ÍNDICE DE DESARROLLO HUMANO. Índices e indicadores de desarrollo humano. 2018. Disponible en: http://hdr.undp.org/sites/default/files/2018_human_development_statistical_update_es.pdf. Acceso en:

LARRAÑAGA, Osvaldo. La medición de la pobreza en dimensiones distintas al ingreso. CEPAL. Serie Estudios estadísticos y prospectivos, n. 58, 2007. Disponible en: https://repositorio.cepal.org/bitstream/ handle/11362/4760/S0700697_es.pdf?sequence=1\&isAllowed=y. Acceso en:

LASAINE, Emma. e-government: a tool for poverty reduction?. Borgen Magazine, 2015. Disponible en: https://www.borgenmagazine.com/e-government-tool/. Acceso en:

LIANG Li; KUI Du; WEI Zhang y JI $\square$ YE Mao. Poverty alleviation through government $\square$ led e $\square$ commerce development in rural China: an activity theory perspective. Information System Journal, 2018. https://doi. $\operatorname{org} / 10.1111 /$ isj.12199.

LUNA-REYES, Luis Felipe. Trust and collaboration in interorganizational information technology projects in the public sector. Gestión y Política Pública, Volumen Temático sobre Gobierno Electrónico, p. 171-210, 2013. Disponible en:http://www.gestionypoliticapublica.cide.edu/VolTem_Gobierno_Electronico/LunaReyes_2013_eng.pdf. Acceso en:

LUNA-REYES, Luis Felipe; GIL-GARCÍA, Ramón, y CELORIO, José Antonio. El mundo del e-gob local: evidencias de municipios digitales en el mundo. Ciudad de México: Infotec I+D+i, 2010.

MÉXICO. Consejo Nacional de Evaluación de la Política de Desarrollo Social. Medición de la pobreza en México y en las entidades federativas. Disponible en: http://www.coneval.gob.mx/Medicion/Documents/Pobreza\%202014_CONEVAL_web.pdf. Acceso en: 04 feb. 2014. 
MÉXICO. Consejo Nacional de Evaluación de la Política de Desarrollo Social. Pobreza en México, medición de la pobreza. Disponible en: http://webdrp.coneval.org.mx/Medicion/MP/Paginas/Pobreza-2018.aspx. Acceso en: 03 feb. 2018.

MÉXICO. Consejo Nacional de Evaluación de la Política de Desarrollo Social. Medición multidimensional de la pobreza en México: un enfoque de bienestar económico y de derechos sociales. https://www.coneval.org.mx/ InformesPublicaciones/FolletosInstitucionales/Documents/Medicion-multidimensional-de-la-pobrezaen-Mexico.pdf. Acceso en: 05 feb. 2019.

MÉXICO. Diario Oficial de la Federación. Disponible en: https:/ /www.dof.gob.mx. 2019. Acceso en:

MÉXICO. Instituto Nacional de Geografía y Estadística. Disponible en: https://www.inegi.org.mx/datos/2018. Acceso en:

MÉXICO. Instituto Federal de Telecomunicaciones. Disponible en: http://www.ift.org.mx/pagina-de-inicio/informes-estadisticos-trimestrales. 2019. Acceso en:

MÉXICO. Encuesta Nacional sobre Disponibilidad y Uso de Tecnologías de la Información en los Hogares (ENDUTIH). 2019. Disponible en: https://www.inegi.org.mx/programas/dutih/2019/. Acceso en:

MISHRA, Alok, y MISHRA, Deepti. E-Government: exploring the different dimensions of challenges, implementation, and success factors. The Data Base for Advances in Information Systems, v. 42, n. 4, p. 23-37, 2011. Disponible en: https://www.researchgate.net/publication/220627546. Acceso en:

MORLAN, Iñaki. Modelo de dinámica de sistemas para la implantación de tecnologías de la información en la gestión estratégica universitaria. Tesis -Universidad del País Vasco / Euskal Herriko Unibertsitatea, 2010.

MUKHOPADHYAY, Sandip; BOUWMAN, Harry y PRASAD, Mahadeo. An open platform centric approach for scalable government service delivery to the poor: The Aadhaar case. Government Information Quarterly, v. 36, n. 3, p. 437-448, 2019. Doi: 10.1016/j.giq.2019.05.001.

MURITALA, Olaseni; ROWLE, Jennifer y JOHNSON, Frances. The multi-dimensional digital divide: perspectives from an e-government portal in Nigeria. Government Information Quarterly, v. 34, n. 2, p. 329-339, 2017. Doi: 10.1016/j.giq.2017.02.002.

MUTULA, Stephen. E-Government's Role in Poverty Alleviation: Case study of South Africa. In: MUTULA, Stephen. Cases on progressions and challenges in ICT utilization for citizen-centric governance. Botswana: University of Botswana, 2013. p. 44-68. Doi: 10.4018/978-1-4666-2071-1.ch003.

OXFORD POVERTY AND HUMAN DEVELOPMENT INITIATIVE; UNITED NATIONS DEVELOPMENT PROGRAMME. Global multidimensional poverty index 2019: illuminating inequalities. 2019. Disponible en: http://hdr.undp.org/sites/default/files/mpi_2019_publication.pdf. Acceso en:

ESTADOS UNIDOS. Programa de las Naciones Unidas para el Desarrollo (PNUD). Informe sobre desarrollo bumano 2019. Disponible en: http://hdr.undp.org/sites/default/files/hdr_2019_overview_-_spanish.pdf. Acceso en:

QUINTERO, Danny y LÓPEZ, Sandra. Análisis estructural: un apoyo para el modelado con dinámica de sistemas. Revista Avances en Sistemas e Informática, v. 7, n. 3, 2010. Disponible en: http:/ /www.bdigital.unal.edu. co/28807/1/26663-93574-1-PB.pdf. Acceso en:

RIVERA, Eugenio. Concepto y problemas de la construcción del gobierno electrónico. Revista Gestión y Politica Pública, CIDE, México, v. 15, n. 2, p. 259-305, 2006.

ROBLEDO, Jorge y FERNANDO, Yony. Estudio de un proceso de innovación utilizando la dinámica de sistemas. Cuadernos de Administración, v. 21, n. 35, p. 127-159, 2008. Disponible en: http://www.scielo.org.co/ pdf/cadm/v21n35/v21n35a06.pdf. Acceso en: 
SANDOVAL, Rodrigo. Explorando la brecha digital en México: diagnóstico del proyecto e-México en el Estado de México. Espacios Públicos, p. 292-306, 2006. Disponible en: http://www.redalyc.org/articulo. oa?id=67601717. Acceso en:

SANJO, Faniran y KAYODE, Olaniyan. e-Governance in Community-Based Poverty Reduction Strategies. Rights link, p. 144-148, 2008. Disponible en: Acceso en:

SANTA, Ricardo; MACDONALD, Jason y FERRER, Mario. The role of trust in e-Government effectiveness, operational effectiveness and user satisfaction: lessons from Saudi Arabia in e-G2B. Government Information Quarterly, v. 36, n. 1, p. 39-50, 2019. Doi: https://doi.org/10.1016/j.giq.2018.10.007.

SAURABH, Chandra. Infrastructure for e-Government Development Issues and Challenges in India. Dynamics of Public Administration, v. 35, n. 1, p. 129-149, 2018. Doi: 10.5958/0976-0733.2018.00010.X.

SAXENA, K.B.C., y CHAHUAN, Kalpana. E-Governance for rural poor: issues \& challenges. FIIB Business Review, v. 2, n. 4, 2013. Doi: 10.1177/2455265820130402.

SEONG-JAE, Min. From the digital divide to the democratic divide: internet skills, political interest, and the second-level digital divide in political internet use. Journal of Information Technology \& Politics, v.7, n. 1, p. 22-35, 2010. DOI: 10.1080/19331680903109402.

SCOTT, John; GUTIÉRREZ, Aida, y CHÁVEZ, Carolina. Medición multidimensional de la pobreza rural en México acceso efectivo y nuevas dimensiones. Instituto de Estudios Peruanos. 2019. Disponible en: http:/ / repositorio.iep.org.pe/handle/IEP/9. Acceso en:

SPENCE, Randy y SMITH, Matthew. ICT, development, and poverty reduction: five emerging stories. Information Technologies \& International Development, v. 6, Special Edition, p. 11-17, 2010.

UNITED NATIONS. Designing e-Government for the Poor. Economic and Social Commission for Asia and The Pacific Asian Development Bank Institute. 2005 Disponible en: https://www.adb.org/sites/default/files/publication/159381/adbi-e-gov-poor.pdf. Acceso en:

UNITED NATIONS. Objetivos de desarrollo sostenible. 2019. Disponible en: https://www.un.org/sustainabledevelopment/es/poverty/. Acceso en:

VALERA, Rogelio y HERNÁNDEZ, Juan Manuel. Pobreza multidimensional y mercado laboral en México. Cuadernos de Economía, v. 39, n. 79, 2019. DOI: 10.15446/cuad.econ.v39n79.71201. 
Para publicar na revista Brasileira de Políticas Públicas, acesse o endereço eletrônico www.rbpp.uniceub.br

Observe as normas de publicação, para facilitar e agilizar o trabalho de edição. 